\title{
Spectral Relaxations and Fair Densest Subgraphs
}

\author{
Aris Anagnostopoulos \\ Sapienza, University of Rome
}

\author{
Luca Becchetti \\ Sapienza, University of Rome
}

\author{
Adriano Fazzone \\ Sapienza, University of Rome
}

\author{
Cristina Menghini \\ Sapienza, University of Rome
}

\begin{abstract}
Reducing hidden bias in the data and ensuring fairness in algorithmic data analysis has recently received significant attention. In this paper, we address the problem of identifying a densest subgraph, while ensuring that none of two protected attributes is disparately impacted.

Unfortunately, the underlying algorithmic problem is NP-hard, even in its approximation version: approximating the densest fair subgraph with a polynomial time algorithm is at least as hard a the densest subgraph problem of at most $k$ vertices, for which no constant approximation algorithms are known.

Despite such negative premises, we are able to provide approximation results in two important cases. In particular, we are able to prove that a suitable spectral embedding allows recovery of an almost optimal, fair, dense subgraph hidden in the input data, whenever one is present, a result that is further supported by experimental evidence. We also show a polynomial time, 2-approximation algorithm, whenever the underlying graph is itself fair. We finally prove that, under the small set expansion hypothesis, this result is tight for fair graphs.

The above theoretical findings drive the design of heuristics, which we experimentally evaluate on a scenario based on real data, in which our aim is striking a good balance between diversity and highly correlated items from Amazon co-purchasing graphs.
\end{abstract}

\section{CCS CONCEPTS}

-Theory of computation $\rightarrow$ Graph algorithms analysis; $•$ Informatio systems $\rightarrow$ Web searching and information discovery;

\section{KEYWORDS}

densest subgraph, fairness, spectral graph analysis

\section{ACM Reference format:}

Aris Anagnostopoulos, Luca Becchetti, Adriano Fazzone, Cristina Menghini, and Chris Schwiegelshohn. 2019. Spectral Relaxations and Fair Densest Subgraphs. In Proceedings of Woodstock '18: ACM Symposium on Neural Gaze Detection, Woodstock, NY, June 03-05, 2018 (Woodstock '18), 11 pages. DOI: $10.1145 / 1122445.1122456$

Permission to make digital or hard copies of all or part of this work for personal or classroom use is granted without fee provided that copies are not made or distributed for profit or commercial advantage and that copies bear this notice and the full citation on the first page. Copyrights for components of this work owned by others than ACM must be honored. Abstracting with credit is permitted. To copy otherwise, or republish, to post on servers or to redistribute to lists, requires prior specific permission and/or a fee. Request permissions from permissions@acm.org.

Woodstock '18, Woodstock, NY

(c) 2019 ACM. 978-1-4503-9999-9/18/06 ..\$15.00

DOI: $10.1145 / 1122445.1122456$

\author{
Chris Schwiegelshohn \\ Sapienza, University of Rome
}

\section{INTRODUCTION}

The identification of dense subgraphs is a fundamental primitive in community detection and graph mining [19, 25, 35, 40, 46]. Given an underlying graph $G=(V, E)$, the density of a node set $S \subseteq V$ is defined as $\frac{2 \cdot|E \cap S \times S|}{|S|}$. In most mining scenarios, communities are assumed to have a high intra-community density versus a lower inter-community density. In this sense, density is arguably the most natural measure of quality for evaluating and comparing communities in graphs (see [12] for an extensive survey.)

In this paper, we consider the densest subgraph problem with fairness constraints. Specifically, we are given a binary labeling of the nodes of the graph $\ell: V \rightarrow\{-1,1\}$. The labeling corresponds to an attribute that ideally should be uncorrelated with community membership. Our goal is to compute a set of nodes $S \subseteq V$ of maximum density while ensuring that $S$ contains an equal number of representatives of either label. The problem has a number of motivating applications, some of which are discussed below.

Mitigation of Polarization. Social networks are very prone to polarization among users [9]: reinforcement of user preferences can lead to feedback loops. For example, recommender systems incentivize disagreement minimization, leading to echo chambers among users with similar preferences. This problem has been considered for example by [34], who studied the problem of identifying a graph of connections between users (of two different opinions), such that polarization and disagreement are simultaneously minimized. The notions behind the fair densest subgraph problem are chosely related: Its goal is to maximize agreement while avoiding polarization ${ }^{1}$.

Team Formation. In crowdsourcing, team formation consists in identifying a set of workers, whose collective skill set incldes all skills that are required for processing some given jobs. Lappas et al. [30] proposed subgraph density as a way of modeling the effectiveness of multiple individuals when working together. The potential benefits of team diversity are well documented in organizational psychology [24] studies and also highlighted by recent work (e.g., see [32] and follow-up work). Diversity in turn can be naturally modeled via fairness constraints.

Diversity in Association Rule Mining. Sozio and Gionis [43] study dense subgraphs for association rule mining: Given a set of tags used to label objects, the densest subgraph problem allows to determine additional related tags that can be used for a better description of the objects. It is common that the tags that are added are semantically identical to those already used. We argue that an appropriate labelling of the tags followed by solving the fair densest

\footnotetext{
${ }^{1}$ The paper by [34] is similar in spirit, but very different in terms of problem modelling.
} 
subgraph problem allows recovery of a set of tags that are not only closely related, but also unique.

Algorithmic Fairness. As pioneered by Chierichetti et al. [14], there has recently been considerable work on clustering data sets using the disparity of impact measure. Conceptually, the aim is to perform data analysis such that the resulting clustering or classifier does not discriminate based on some protected attribute. In our case, finding a densest subgraph such that a protected attribute is not disparately impacted is equivalent to the definition of the fair densest subgraph problem.

\subsection{Contributions}

As it turns out (see Section 3), the fair densest subgraph problem is intractable in general, while its unconstrained counterpart can be solved optimally through network flow [22]. Nevertheless, we have some quantifiable results regarding approximation algorithms in special cases. If the underlying graph itself is fair, we can show that there exists a 2-approximation algorithm. We further show that, assuming the widely used small set expansion hypothesis [38], this is the best possible. We also consider the case where the graph itself is not fair and we instead aim for a proportional representation. For this, in our opinion more flexible variant of the problem, we show that the results for fair graphs can be extended.

Although this worst-case behavior is discouraging, the possibility of effective algorithms is not ruled out on practical instances. To this end, we identify properties that, if satisfied by some subgraph of the network under consideration, will afford recovery of an approximately fair, dense subgraph. More precisely, our goal in this respect is designing a heuristic that

(a) has a quantifiable guarantee if the underlying graph is well-behaved and

(b) is practically viable.

Our main result is a spectral algorithm that satisfies both of these requirements. In particular, the practical viability of our algorithm underscores that our notion of a well-behaved graph is a realistic one. As a candidate application, we considered the scenario of providing diverse recommendations of high quality, using data from the Amazon product co-purchasing graph. Our experiments not only confirm the quality of the output solutions, but also the scalability of our approach, which may not be the case for a conventional combinatorial approximation algorithm.

Overview of approach. Our approach builds on the finding [27, 33] that the densest subgraph problem admits a spectral formulation. Specifically, an approximate densest subgraph can be computed by selecting nodes for inclusion according to the magnitudes of the corresponding entries in the main eigenvector of $G$ 's adjacency matrix. Unfortunately, this approach does not afford balanced solutions in general. In a nutshell, we sidestep this issue by first projecting the adjacency matrix onto a suitable "fair" subspace, an operation that corresponds to the enforcement of "soft" fairness constraints.

To see why the conventional spectral approach of [27] may not work $^{2}$ and why our approach mitigates the issue, Figure 1 presents

${ }^{2}$ In fact, this applies to any approach based on unconstrained maximization of the induced subgraph's density. plots obtained from Amazon books on US politics [29]. The books are labeled as either conservative or liberal, which corresponds to the labels -1 or 1 . As described above, a candidate application may be to find a selection of books that are of interest to multiple readers, while mitigating potential polarization along political lines.

On the left, we observe the books ordered according to their corresponding entries in the main eigenvector of the adjacency matrix of the co-purchase graph. Books are also colored according to political orientation. We can observe that, whereas liberal books are well distributed, conservative ones are clustered. On the right we observe the results after application of our spectral embedding, which affords recovery of a subgraph of the co-purchase graph that is both dense and approximately balanced. Note that now conservative books are also well distributed along the principal component.

\subsection{Related work}

Densest Subgraph. Identifying dense subgraphs is a key primitive in a number of applications; see [18, 20,21, 47]. The problem can be solved optimally in polynomial time [22]. On the contrary, the fair densest subgraph problem is highly related to the densest subgraph problem limited to at most $k$ nodes, which cannot be approximated up to a factor of $n^{1 /(\log \log n)^{c}}$ for some $c>0$ assuming the exponential time hypothesis [31] and for which state-of-the-art methods yield an $O\left(n^{1 / 4+\varepsilon}\right)$ approximation [6].

Algorithmic Fairness. Fairness in algorithms received considerable attention in the recent past, see $[23,45,48,50]$ and references therein. The closely related notion of disparate impact was first proposed by [17]. It has since been used by [49] and Noriega-Campero et al. [37] for classification and Celis et al. [10,11] for voting and ranking problems. Another problem that received considerable attention is fair clustering. This was first proposed as a problem by [14] in the case of a binary protected attribute. It was then investigated for various objectives and more color classes in theirs and subsequent work [1, 4, 5, 26, 39, 42].

Most closely related to our work are the papers by [28, 41, 44]. The former two papers considers the problem of executing a principal component analysis in a fair manner. Specifically, given a matrix $A$ where the rows are colored (e.g. every row corresponds to a man or a woman), they ask for an algorithm that finds the finds a rank $k$ matrix $A^{\prime}$ whose residual error $\left\|A-A^{\prime}\right\|$ is small for both types of rows simultaneously. While our method is similarly based on using the principal component in a fair manner, the difference is that we may be forced to treat the classes differently, if we aim to uncover a dense subgraph as illustrated in the example mentioned above and in Figure 1.

The latter paper by [28] considers spectral clustering problems such as normalized cut. Like our work, they project the Laplacian matrix of a graph $G$ onto a suitable "fair" subspace, and then run $k$-means on the subspace spanned by the smallest resulting eigenvectors. Under a fair version of the stochastic block model, they show that this algorithm recovers planted fair partitions. Our work continues this idea by applying the technique to the densest subgraph problem. 

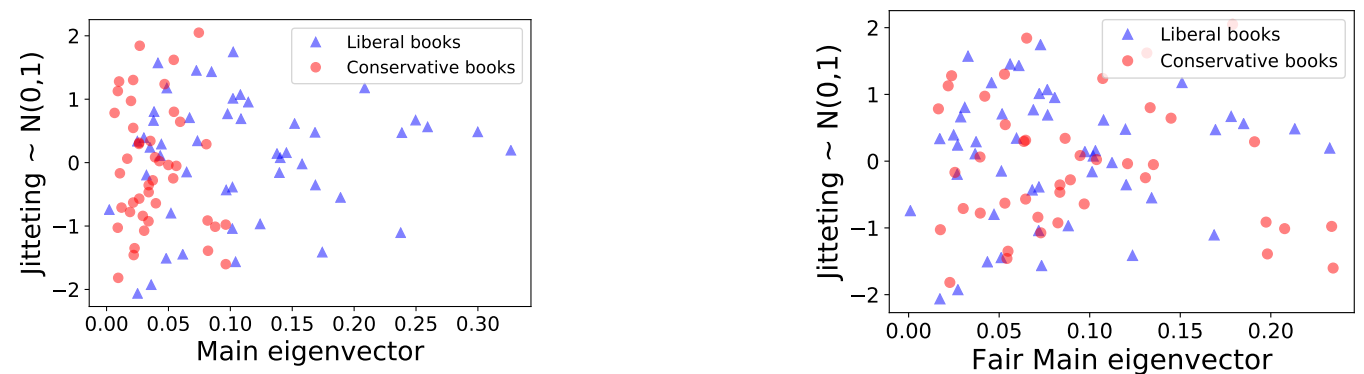

Figure 1: Projection of books (see Section 4) onto the first principal component. (Left) Original data. (Right) Data after spectral embedding. Books are ordered on the $x$ axis according to their corresponding entries in the main eigenvector, whereas on the $y$ axis we have random noise for visualization.

\subsection{Preliminaries and Notation}

We consider undirected graphs $G(V, E, w)$, where $V$ is the set of $n$ nodes, $E \subset V \times V$ is the set of edges, and $w: E \rightarrow \mathbb{R}_{\geq 0}$ is a weight function. We denote the (weighted) adjacency matrix of $G$ by $A$. For a subset $E^{\prime} \subset E$ of the edges, we let $w\left(E^{\prime}\right)=\sum_{e \in E^{\prime}} w(e)$. Considered $u \in V$, its (weighted) degree is $d_{u}=\sum_{e \cap\{v\} \neq \emptyset} w(e){ }^{3}$ We also let $d_{\max }=\max _{u} d_{u}$. Considered $S \subseteq V$, we denote by $G_{S}$ the induced subgraph. The density $D_{S}(G)$ of $S \subseteq V$ is simply the average degree of $G_{S}$, namely: namely:

$$
D_{S}(G)=\frac{2 \cdot|E \cap S \times S|}{|S|} .
$$

We omit $G$ from $D_{S}(G)$, whenever clear from context.

A coloring of the vertices is simply a map $c: V \rightarrow[\ell]$ of $V$, where $[\ell]:=\{1,2, \ldots, \ell\}$. A set $S \subset V$ is called fair if $\mid S \cap\{v \in V \mid c(v)=$ $1\}|=| S \cap\{v \in V \mid c(v)=2\}|=\cdots=| S \cap\{v \in V \mid c(v)=\ell\} \mid$. A graph is called fair if $V$ is fair. In the remainder, we provide positive results for the important case $\ell=2$. In this case, for simplicity of exposition we denote the colors red and blue and we use Red $:=\{v \in V \mid c(v)=$ red $\}$ and Blue $:=\{v \in V \mid c(v)=$ blue $\}$ to refer to nodes of the respective color.

Definition 1.1 (Fair Densest Subgraph Problem). Given a (weighted) graph $G(V, E, w)$ and a coloring $c$ of its vertices, identify a fair subset $S \subseteq V$ that maximizes $D_{S}$.

The fair densest subgraph problem is obviously a constrained version of the densest subgraph problem. It turns out to be considerably harder than its (polynomially solvable) unconstrained counterpart, as we show in Section 3.

Linear algebra notation. We denote by $\lambda_{1} \geq \lambda_{2} \geq \ldots \geq \lambda_{n}$ the eigenvalues of $A$ and by $v_{i}$ its $i$-th eigenvector. We also set $\lambda=\max _{i>2}\left(\lambda_{2},\left|\lambda_{n}\right|\right)$. Note that we always have $\lambda_{1} \leq d_{\text {max }}$. For a subset $S \subset V$, we denote by $\chi$ its normalized indicator vector, where $S$ is understood from context. Namely, $\chi_{i}=1 / \sqrt{|S|}$ if $i \in S, \chi_{i}=0$ otherwise. Finally, for a vector $x \in \mathbb{R}^{n}$, we let $\|x\|=\sqrt{\sum_{i=1}^{n} x_{i}^{2}}$, the 2-norm of $x$.

\footnotetext{
${ }^{3}$ The term volume is often used rather than weighted degree. Here we simply use the term "degree" liberally, since our algorithms and results equally apply to unweighted and weighted graphs.

${ }^{4}$ The right-hand side becomes $\frac{2 w(E \cap S \times S)}{|S|}$ for weighted graphs.
}

\section{SPECTRAL RELAXATIONS FOR THE FAIR DENSEST SUBGRAPH}

As observed in Kannan and Vinay [27], the densest subgraph problem admits a spectral formulation. In particular, denoted by $x$ an indicator vector over the vertex set, the indicator vector of the vertex subset maxizing density is the maximizer of the following expression:

$$
\max _{x \in\{0,1\}^{n}} \frac{x^{T} A x}{x^{T} x} .
$$

Now, assume that each node is colored with one of two colors, red or blue. The optimal solution $x^{*}$ might well overrepresent one of the colors. To formulate the problem of computing a fair solution, we can add the constraint

$$
\Leftrightarrow \quad \sum_{\text {node } i \text { is red }} x_{i}=\sum_{\text {is red }} x_{i}-\sum_{\text {node } i \text { is blue }} x_{i} x_{\text {is blue }} x_{i}=0 .
$$

If we define the (unit 2-norm) vector

$$
f_{i}= \begin{cases}\frac{1}{\sqrt{n}} & \text { if node } i \text { is red } \\ -\frac{1}{\sqrt{n}} & \text { if node } i \text { is blue, }\end{cases}
$$

the above constraint can be described as $f^{T} x=0$. We call such an $x$ fair. Conversely, very unbiased solutions will have high inner products with $f$.

Fair Densest Subgraph: Spectral Relaxation. Based on the considerations above, our approach transforms the input data (in this case the adjacency matrix $A$ ) by first projecting them onto the kernel of $f$. Namely, we first consider the following formulation of the fair densest subgraph problem:

$$
\max _{x \in\{0,1\}^{n}} \frac{2 x^{T}\left(I-f f^{T}\right) A\left(I-f f^{T}\right) x}{x^{T} x} .
$$

It should be noted that, for any fair subset $S$ with indicator $x$, we have $\frac{2 x^{T} A x}{x^{T} x}=\frac{2 x^{T}\left(I-f f^{T}\right) A\left(I-f f^{T}\right) x}{x^{T} x}$. Conversely, for any indicator vector $x \notin \operatorname{span}\left(I-f f^{T}\right)$, the objective value can only decrease.

We next note that by relaxing $x$ to be an arbitrary vector, the above expression is maximized by the main eigenvector of ( $I-$ 
$\left.f f^{T}\right) A\left(I-f f^{T}\right)$. Indeed, [27] established a relationship between the first eigenvector of the adjacency matrix $A$ and an approximately densest subgraph. Similar ideas are also implicit in the work of [33]. The above relaxation corresponds to replacing hard fairness constraints with soft ones.

It is straightforward to encode more complicated fairness constraints using this technique. Suppose, for example, that we are given $\ell$ colors, and wish to output a subgraph such that every color is featured equally often. This induces a set of constraints

$$
\begin{aligned}
\sum_{\text {node } i \text { is red }} x_{i} & =\sum_{\text {node } i \text { is blue }} x_{i} \\
\sum_{\text {node } i \text { is red }} x_{i} & =\sum_{\text {node } i \text { is green }} x_{i} \\
& \vdots \\
\sum_{\text {node } i \text { is red }} x_{i} & =\sum_{\text {node } i \text { is yellow }} x_{i}
\end{aligned}
$$

The vectors satisfying all of these constraints lie in the nullspace of some $\ell-1$ dimensional subspace $S$. Assume that $F$ is a matrix such that the columns of $F$ form an orthogonal basis of $S$. Then the above technique leads to the problem

$$
\max _{x \in\{0,1\}^{n}} \frac{2 x^{T}\left(I-F F^{T}\right) A\left(I-F F^{T}\right) x}{x^{T} x} .
$$

More generally, this technique can be extended to any system of linear constraints. Only has to merely find a suitable basis and project $A$ onto said basis.

We note that while the technique can handle these more complicated constraints, leveraging this in an algorithm with provable guarantees seems very difficult. Nevertheless, our experiments dealing with multiple colors showcase that we can still tackle more complicated fairness constraints with success in practice, see Section 4.1 .

\subsection{Recovery of Dense Fair Subgraphs in Almost Regular Graphs}

To prove our main result we need the following definition:

Definition 2.1. Graph $H=\left(V_{H}, E_{H}\right)$ is $(d, \epsilon)$-regular if a $d$ exists, such that $(1-\epsilon) d \leq d_{i} \leq(1+\epsilon) d$, for every $i \in V_{H}$.

Theorem 2.2. Assume we have a graph $G=(V, E, w)$ with a 2-coloring of the nodes. Assume the spectrum of $A$ satisfies $\lambda_{1} \geq 4 \lambda^{5}$ Assume further that $G$ contains a fair subset $S$ such that: (1) $G_{S}$ is $(d, \epsilon)$-regular and $(2) d \geq(1-\theta) d_{\max }$. In this case, it is possible to recover all but $16(\epsilon+\theta)|S|$ of the vertices in $S$ in polynomial time.

Intuitively, the result above states that, if the underlying network $G$ is an expander containing an almost-regular, dense and fair subgraph, we can approximately retrieve it in polynomial time. Succintly, this follows because, under these assumptions, the indicator vector of $S$ forms a small angle with the main eigenvector of $\left(I-f f^{T}\right) A\left(I-f f^{T}\right)$.

Proof of Theorem 2.2. In the remainder of this proof, we denote by $\hat{\lambda}_{1} \geq, \ldots, \geq \hat{\lambda}_{n}$ the eigenvalues of $\left(I-f f^{T}\right) A\left(I-f f^{T}\right)$

\footnotetext{
${ }^{5}$ That is, $G$ is an expander.
}

and by $\hat{v}_{i}$ the $i$-th associated eigenvector. For a vertex $i$ of $G_{S}$ we denote by $\hat{d}_{i}$ its degree in $G_{S}$. We denote by $\chi$ the indicator vector of $S$ and we let $m=|S|$.

As a first step, we summarize straightforward, yet useful properties of the spectrum of $\left(I-f f^{T}\right) A\left(I-f f^{T}\right)$.

Claim 1. Whenever $\hat{\lambda}_{i} \neq 0$ we have:

$$
\left(I-f f^{T}\right) \hat{v}_{i}=\hat{v}_{i} \text { and } \hat{\lambda}_{i}=\hat{v}_{i}^{T} A \hat{v}_{i}
$$

Proof. If $\hat{\lambda}_{i} \neq 0$, we have:

$$
\left(I-f f^{T}\right) A\left(I-f f^{T}\right) \hat{v}_{i}=\hat{\lambda}_{i} \hat{v}_{i} .
$$

Since $\left(I-f f^{T}\right)$ is a projection matrix, if we pre-multiply both members of the above equation by $\left(I-f f^{T}\right)$ we have:

$$
\left(I-f f^{T}\right) A\left(I-f f^{T}\right) \hat{v}_{i}=\hat{\lambda}_{i}\left(I-f f^{T}\right) \hat{v}_{i} .
$$

Subtracting the first equation from the second and recalling that $\hat{\lambda}_{i} \neq 0$ immediately the first claim.

The second claim follows immediately from the first:

$$
\hat{\lambda}_{i}=\hat{v}_{i}^{T}\left(I-f f^{T}\right) A\left(I-f f^{T}\right) \hat{v}_{i}=\hat{v}_{i}^{T} A \hat{v}_{i} .
$$

It should be noted that, as a consequence of Claim 1, we always have:

$$
\hat{\lambda}_{1}=\hat{v}_{1}^{T}\left(I-f f^{T}\right) A\left(I-f f^{T}\right) \hat{v}_{1}=\hat{v}_{1}^{T} A \hat{v}_{1} \leq v_{1}^{T} A v_{1}=\lambda_{1} .
$$

Note that this last property does not apply to the other eigenvalues in general. The first important, technical step to prove Theorem 2.2 is showing that the hypothesis $\lambda_{1} \geq 4 \lambda$ implies that $\hat{\lambda}_{2}$ cannot be "too large".

Lemma 2.3. Assume the spectrum of A satisfies the condition $\lambda_{1} \geq$ $4 \lambda_{2}$. Then $\hat{\lambda}_{2} \leq \frac{3}{4} \lambda_{1}$.

Proof. We first express $\hat{v}_{2}$ as $\hat{v}_{2}=\gamma v_{1}+z$, where $z$ is $\hat{v}_{2}$ 's component orthogonal to $v_{1}$, the main eigenvector of $A$. Note that, since $v_{1}$ has unit norm, we have $\gamma^{2}+\|z\|^{2}=1$. Next:

$$
\hat{\lambda}_{2}=\left(\gamma v_{1}+z\right)^{T} A\left(\gamma v_{1}+z\right)^{T}=\gamma \lambda_{1}+z^{T} A z,
$$

where the first equality follows from Claim 1, while the second follows since $z \in \operatorname{span}\left(v_{2}, \ldots, v_{n}\right)$ by definition and the $v_{i}$ 's form an orthonormal basis. Next, assume $\gamma \geq 1 / 2$. In this case, we have:

$$
\hat{\lambda}_{2}=\gamma \lambda_{1}+z^{T} A z=\gamma \lambda_{1}+\|z\|^{2} \frac{z^{T} A z}{\|z\|^{2}} \geq \frac{3}{8} \lambda_{1} .
$$

Here, the third inequality follows since i) $\|z\|^{2}=1-\gamma^{2} \leq 1 / 2$, while $z \in \operatorname{span}\left(v_{2}, \ldots, v_{n}\right)$ implies:

$$
\left|\frac{z^{T} A z}{\|z\|^{2}}\right| \leq \max _{w \perp v_{1}}\left|\frac{w^{T} A w}{\|w\|^{2}}\right|=\lambda .
$$

But (3) contradicts our assumption that $\lambda_{1} \geq 4 \lambda$. On the other hand, if $\gamma \leq 1 / 2$, (2) implies:

$$
\begin{aligned}
& \hat{\lambda}_{2}=\gamma \lambda_{1}+z^{T} A z \leq \gamma \lambda_{1}+z^{T} A z \\
& \leq \gamma \lambda_{1}+\|z\|^{2} \max _{w \perp v_{1}} \frac{w^{T} A w}{\|w\|^{2}} \\
& =\gamma \lambda_{1}+\left(1-\gamma^{2}\right) \lambda_{2} \leq \frac{\lambda_{1}}{2}+\lambda_{2} \leq \frac{3}{4} \lambda_{1} .
\end{aligned}
$$


The second step is showing that Lemma 2.3 implies that the indicator vector of the fair densest subgraph is close to $\hat{v}_{1}$ :

Lemma 2.4. Assume the hypotheses of Theorem 2.2 hold. Then:

$$
\left\|\chi-\hat{v}_{1}\right\|^{2} \leq 4(\epsilon+\theta) .
$$

Proof. We begin by noting that $\chi^{T} f=0$ by definition, which implies $\left(I-f f^{T}\right) \chi=\chi$. We therefore have:

$$
\chi^{T}\left(I-f f^{T}\right) A\left(I-f f^{T}\right) \chi=\frac{\sum_{i \in S} \hat{d}_{i}}{m} \geq(1-\epsilon) d,
$$

Next, we decompose $\chi$ along its components respectively parallel and orthogonal to $\hat{v}_{1}$, namely, $\chi=\alpha \hat{v}_{1}+z$, and we note that $\|z\|^{2}=1-\alpha^{2}$, since both $\hat{v}_{1}$ and $\chi$ are unit norm vectors. Set $B=\left(I-f f^{T}\right) A\left(I-f f^{T}\right)$ for the sake of space. We have:

$$
\begin{aligned}
& \chi^{T} B \chi=\left(\alpha \hat{v}_{1}+z\right)^{T} B s\left(\alpha \hat{v}_{1}+z\right)=\alpha^{2} \hat{\lambda}_{1}+z^{T} B z \\
& \leq \alpha^{2} \hat{\lambda}_{1}+\hat{\lambda}_{2}\|z\|^{2} \leq \alpha^{2} \hat{\lambda}_{1}+\left(1-\alpha^{2}\right) \hat{\lambda}_{2} .
\end{aligned}
$$

Putting together (4) and (5) yields $\alpha^{2} \geq \frac{(1-\epsilon) d-\hat{\lambda}_{2}}{\hat{\lambda}_{1}-\hat{\lambda}_{2}}$. Now:

$$
\begin{aligned}
& \|\chi-v\|^{2} \leq 1-\frac{(1-\epsilon) d-\hat{\lambda}_{2}}{\hat{\lambda}_{1}-\hat{\lambda}_{2}} \\
& \leq 1-\frac{(1-\epsilon)(1-\theta) d_{\max }-\hat{\lambda}_{2}}{\lambda_{1}-\hat{\lambda}_{2}} \\
& \leq 1-\frac{(1-\epsilon)(1-\theta) \lambda_{1}-\hat{\lambda}_{2}}{\lambda_{1}-\hat{\lambda}_{2}} \\
& <1-\frac{\lambda_{1}-\hat{\lambda}_{2}-(\epsilon+\theta) \lambda_{1}}{\lambda_{1}-\hat{\lambda}_{2}} \\
& =\frac{(\epsilon+\theta) \lambda_{1}}{\lambda_{1}-\hat{\lambda}_{2}} \leq 4(\epsilon+\theta) .
\end{aligned}
$$

Here, the second inequality follows from our hypotheses on $d$ and since $\hat{\lambda}_{1} \leq \hat{\lambda}$, the third inequality follows since the main eigenvalue of an adjacency matrix is upper-bounded by the maximum degree of the underlying graph, while the last inequality follows from Lemma 2.3.

COROLlary 2.5. Under the hypotheses of Lemma 2.4, for all but at most $16 m(\epsilon+\theta)$ vertices in $V$ we have: i) $\hat{v}_{1}(i) \geq \frac{1}{2 \sqrt{m}}$ if $i \in S$, ii) $\hat{v}_{1}(i)<\frac{1}{2 \sqrt{m}}$ otherwise.

The algorithm. Our algorithm is based on a sweep of $\hat{v}_{1}[27,33]$ In particular, we run Algorithm GSA (see Algorithm 1) with $M=$ $\left(I-f f^{T}\right) A\left(I-f f^{T}\right)$ and $\Delta=16(\epsilon+\theta)$.

Corollary 2.5 ensures that i) the above algorithm always returns a solution, ii) the solution returned by the algorithm will not be worse than the one obtained by picking $i$ if $\hat{v}_{1}(i) \geq \frac{1}{2 \sqrt{m}}$ and rejecting it otherwise. This concludes the proof of Theorem 2.2.

The running time of the algorithm is dominated by computing the first eigenvector and the projecting of the rows of the Laplacian onto said eigenvector. This can be done, up to $(1+\varepsilon)$ precision, in linear time.

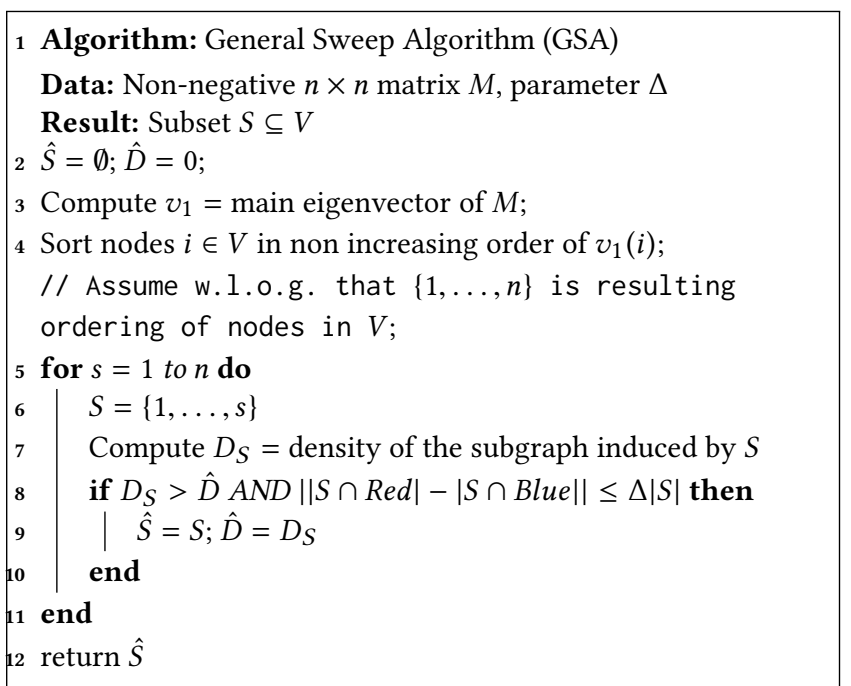

Algorithm 1: General Sweep Algorithm (non-increasing).

\section{HARD CONSTRAINTS AND HARDNESS OF APPROXIMATION}

In general, enforcing fairness can make an "easy" problem intractable and this is the case for the densest subgraph problem. In this context, spectral relaxations can be regarded as a way to mitigate this issue, by enforcing soft fairness constraints to virtually any problem that is amenable to an algebraic formulation.

Nevertheless, in some cases it might be important to assess the price of fairness, by comparing the achievable quality of fair solutions to that of solutions for the original, unconstrained problem. In this section, we complement our algorithmic treatment of fairness with hardness results and approximation algorithms for specific cases. Proofs are omitted for the sake of space, but they are available as supplementary material. Some of our hardness results are based on the small set expansion hypothesis, which we now describe.

Consider a $d$-regular weighted graph $G$ and, for every $S \subset V$, denote by $\Phi(S)$ the expansion ${ }^{6}$ of $S$ [38]. Given two constants $\delta, \eta \in(0,1)$, the small set expansion problem [38] $\operatorname{SSE}(\delta, \eta)$ asks to distinguish between the following two cases:

Completeness There exists a set of nodes $S \subset V$ of size $\delta \cdot|V|$ such that $\Phi(S) \leq \eta$.

Soundness For every set of nodes $S \subset V$ of size $\delta \cdot|V|$, $\Phi(S) \geq 1-\eta$.

Our hardness proofs are based on the small set expansion hypothesis defined as follows.

CONJecture $3.1(\mathrm{SSEH})$. For every $\eta>0$ there exists a $\delta:=$ $\delta(\eta)>0$ such that $\operatorname{SSE}(\eta, \delta)$ is NP-hard.

Recall from Section 1.2 that, whereas the densest subgraph problem is polynomially solvable, the best approximation for the densest at-most- $k$ subgraph problem is in $O\left(n^{1 / 4}\right)$ [7] and cannot be approximated up to a factor of $n^{1 /(\log \log n)^{c}}$ for some $c>0$ assuming the exponential time hypothesis [31] . The next theorem implies

${ }^{6}$ Or conductance. 
that these inapproximability results for the densest at-most- $k$ subgraph problem hold also for the fair densest subgraph problem, showing that fairness constraints can drastically affect hardness of this problem.

TheOREM 3.2. The densest fair subgraph problem is at least as hard as the densest at most $k$ subgraph problem. Moreover, any $\alpha$-approximation to the densest at-most- $k$ subgraph is a $2 \alpha$ approximation to densest fair subgraph.

Proof. Consider an arbitrary graph $G(V, E)$. We consider $V$ to be colored red. Add $k$ blue nodes with no edges. Then the density of the fair densest subgraph is, up to a multiplicative factor of exactly $\frac{1}{2}$, equal to the density of the densest at most $2 k$ subgraph. Conversely, running an algorithm for densest $k$ subgraph with $k=\min (|B l u e|, \mid$ Red $\mid)$, and balancing out the resulting subgraph in post processing decreases the density by at most a factor 2 . (This latter part is explained in more detail in the following theorem).

When the input graph $G$ is itself fair, we can provide stronger bounds.

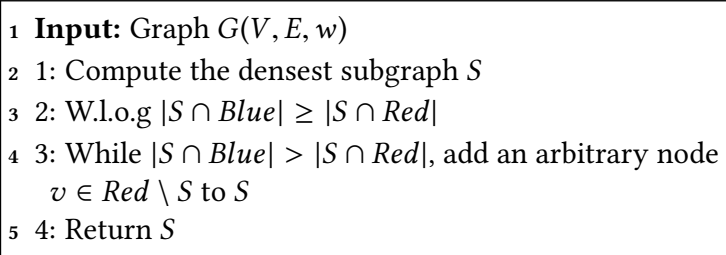

Algorithm 2: Approximate Fair Densest Subgraph

Theorem 3.3. Given a fair graph $G(V, E, w)$, Algorithm 2 computes a fair set $S \subset V$, such that $D_{S} \cdot 2 \geq O P T$, where OPT is the density of the fair densest subgraph.

Proof. We refer to the set $S$ computed after line 1 , and 3 as $S_{1}$ an $S_{2}$, respectively. Since $S_{1}$ is the unconstrained densest subgraph, $D_{S_{1}}>O P T$. For $S_{2}$, we observe that $\left|S_{2}\right| \leq S_{1}+\mid S_{1} \cap$ Blue $|-| S_{1} \cap$ Red $|\leq 2 \cdot| S_{1} \mid$, hence $D_{S_{2}}=\frac{w\left(E_{S_{2}}\right)}{\left|S_{2}\right|} \geq \frac{w\left(E_{S_{1}}\right)}{2\left|S_{1}\right|} \geq \frac{O P T}{2}$.

The running times of both algorithms depend on the running time of the subroutines used to compute dense subgraphs. Unconstrained dense subgraphs can be found by solving a linear program or by computing a max flow $[13,22]$. A faster $(1+\varepsilon)$ approximation that runs in time $O(n \operatorname{polylog}(n))$ also exits $[2,15]$.

For the densest $k$ subgraph problem, the currently best algorithm that computes an $O\left(n^{1 / 4+\varepsilon}\right)$ approximation runs in time $n^{O(1 / \varepsilon)}$ [6]

We conclude this section by showing that approximating the fair densest subgraph problem beyond a factor of 2 is at least as hard as solving $\operatorname{SSE}(\eta, \delta)$. Therefore, barring a major algorithmic breakthrough, Algorithm 2 is optimal. The proof is provided as supplementary material and it is based on the following idea: In regular graphs, for a given set of nodes $S$, the expansion $\Phi(S)$ is related to the density of $S$. We can use this, so that, given a graph $G$, we can carefully construct a colored graph $G^{\prime}$ such that finding the optimal fair densest subgraph in $G^{\prime}$ gives an estimate of the largest-expansion node set in $G$.
THEOREM 3.4. If SSEH holds, computing $a(2-\varepsilon)$ approximation of the fair densest subgraph problem in fair graphs is NP-hard for any $\varepsilon>0$.

Proof. We consider the $S S E(\eta, \delta)$ problem, i.e. let $G(V, E, w)$ be a $d$-regular graph and let $\eta \in(0,1)$ and $\delta=\delta(\eta) \in(0,1 / 2]$ be constants that we will specify later. For any set $S \subset V$ of size $s:=\delta \cdot|V|$, we have $w\left(E_{S}\right):=d \cdot s-\Phi(S) \cdot d \cdot s$.

We construct a colored graph $G^{\prime}\left(V^{\prime}, E^{\prime}, w^{\prime}\right)$ by considering all nodes of $G$ to be colored red, and by adding $|V|$ blue nodes. Of these nodes, we select an arbitrary but fixed subset of $\delta \cdot|V|$ blue nodes that we denote by $B$. Each edge in $E_{B}$ is weighted uniformly by $t:=\frac{2 \cdot d}{s-1}$. The remaining edges are weighted with 0 .

Denote the size of the fair densest subgraph $C$ by $k$. Further, let $C_{\text {red }}=C \cap$ Red. We will distinguish between four basic cases: (1) $k<2 \mu \cdot s$, (2) $2 \mu \cdot s \leq k<2 \cdot s$, (3) $2 \cdot s \leq k<\frac{2}{\mu} s$, and (4) $\frac{2}{\mu} s \leq k$, where $\mu>0$ is suitably small constant specified later. We note that the cases (1) and (4) and (2) and (3) will turn out to be somewhat symmetric, even if slightly different proofs are required in every case.

First, let $k<2 \mu \cdot s$ and again let $B_{k}$ be an arbitrary subset of $B$ of size $k$. Then

$$
D_{C_{r e d} \cup B_{k}} \leq \frac{d \cdot k+w\left(B_{k}\right)}{2 \cdot k} \leq(1+2 \mu) \frac{d}{2},
$$

where the first inequality holds due to regularity.

Now, let $2 \mu \cdot s \leq k<2 \cdot s$. We have

$$
D_{C_{r e d} \cup B_{k}} \leq \frac{\eta \cdot d \cdot s+w\left(B_{k}\right)}{2 \cdot k} \leq\left(1+\frac{2 \eta}{\mu}\right) \frac{d}{2} .
$$

Now, let $2 \cdot s \leq k \leq \frac{2}{\mu} \cdot s$. We will first show that

$$
w(C) \leq \frac{2}{\mu} \cdot \eta \cdot d \cdot k .
$$

For the sake of contradiction, assume that this is not the case. The argument revolves around double counting $w(C)$. There exist $\left(\begin{array}{l}k \\ s\end{array}\right)$ subsets of size $s$ of $C$. Observe that for any such subset $S^{\prime}$ has weight $w\left(S^{\prime}\right) \leq \eta \cdot d \cdot s$ and hence

$$
\sum_{S^{\prime} \subset C \wedge\left|S^{\prime}\right|=s} w\left(S^{\prime}\right) \leq \eta \cdot d \cdot s \cdot\left(\begin{array}{l}
k \\
s
\end{array}\right) .
$$

At the same time, every (possibly 0 valued) edge appears in $\left(\begin{array}{l}k-2 \\ s-2\end{array}\right)$ of these subsets. Hence

$$
\sum_{S^{\prime} \subset C \wedge\left|S^{\prime}\right|=s} w\left(S^{\prime}\right)=w(C) \cdot\left(\begin{array}{l}
k-2 \\
s-2
\end{array}\right)>\frac{2}{\mu} \cdot \eta \cdot d \cdot k \cdot\left(\begin{array}{l}
k-2 \\
s-2
\end{array}\right) .
$$

Combining both equations, we have

$$
\begin{array}{r}
\frac{2}{\mu} \cdot \eta \cdot d \cdot k \cdot\left(\begin{array}{l}
k-2 \\
s-2
\end{array}\right)<\eta \cdot d \cdot s \cdot\left(\begin{array}{l}
k \\
s
\end{array}\right) \\
\Leftrightarrow \frac{2}{\mu}<\frac{k \cdot(k-1)}{s \cdot(s-1)} \frac{s}{k} \leq \frac{2}{\mu},
\end{array}
$$

which is a contradiction.

Consider now the density of any fair cut containing $C \cup B_{k}$, where $B_{k}$ contains $B$ and $k-s$ further arbitrary blue nodes. We 
have

$$
D_{C_{r e d} \cup B_{k}} \leq \frac{\frac{2 \eta}{\mu} \cdot d \cdot k+t \cdot\left(\begin{array}{l}
s \\
2
\end{array}\right)}{2 \cdot k} \leq\left(1+\frac{2 \eta}{\mu}\right) \cdot \frac{d}{2} .
$$

Finally, consider the case $k>\frac{2}{\mu} s$. Then the density of any fair cut containing $C \cup B_{k}$, where $B_{k}$ contains $B$ and $k-s$ further arbitrary blue nodes, is

$$
D_{C_{r e d} \cup B_{k}} \leq \frac{d \cdot k+t \cdot\left(\begin{array}{l}
s \\
2
\end{array}\right)}{2 \cdot k} \leq(1+2 \mu) \frac{d}{2} .
$$

We note that bounds from Equations 6 and 9 and Equations 7 and 10 are identical. For $\varepsilon<\frac{1}{4}$, we set $\mu=\frac{\varepsilon}{2}, \eta \leq \frac{8}{3} \cdot \varepsilon^{2}$. Then the ratio between the terms ?? and 6 and the terms ?? and 7 is at least $2-\varepsilon$. Therefore, approximating the fair densest subgraph problem beyond a factor of 2 solves the $S S E(\eta, \delta)$ problem.

\section{EXPERIMENTAL ANALYSIS}

Worst case bounds are often uninformative when compared with empirical behavior. Algorithm 2 is (assuming that the underlying graph is fair) theoretically optimal and therefore superior to the spectral recovery schemes. As we now describe, the empirical performance between these approaches paints the opposite picture.

Overview. To test the performances of our algorithms on real data we used two publicly available dataset: PoLBooks [29] and AmAzon products metadata [36]. Both (explicitly or implicitly) contain undirected unweighted graphs, whose nodes are products from the Amazon catalog, while an edge between two nodes exists if the corresponding products are frequently co-purchased by the same buyer. Moreover, for both datasets, each product belongs to exactly one category.

We tested our methods in a scenario in which, given a (not necessary fair) labeled graph, our only interest lies in finding fair subgraphs with high density. In this context, we are considering the density of the provided solution as a quality indicator: the higher the density, the better the quality of a solution.

For our experiments we used an Intel Xeon $2.4 \mathrm{GHz}$ with $24 \mathrm{~GB}$ of RAM running Linux Ubuntu 18.04 LTS. All methods have been implemented in Python3 using the functionalities provided by NetworkX ${ }^{7}$ and SciPy ${ }^{8}$ libraries.

Datasets. The PoLBooks data set [29] is an undirected unweighted graph $^{9}$, whose nodes represent books on US politics included in the Amazon catalog, while an edge between two books exists if both books are frequently co-purchased by the same buyer. Each book is further labeled depending on its political stance, possible labels being "liberal", "neutral", and "conservative". For our experiments, we considered only the subgraph induced by "liberal" and "conservative" books, obtaining 92 nodes (49 of which were associated with a "conservative" worldview, 43 with a "liberal" worldview) for 362 edges in total.

The AmAzon products metadata dataset [36] contains descriptions for 15.5 million Amazon products ${ }^{10}$. For a single product,

\footnotetext{
${ }^{7}$ https://networkx.github.io/documentation/stable

${ }^{8}$ https://www.scipy.org

${ }^{9} \mathrm{http}: / /$ www.casos.cs.cmu.edu/computational_tools/

datasets/external/polbooks/polbooks.gml.

${ }^{10}$ https://nijianmo.github.io/amazon/index.html
}

we only considered the product id ( $a \sin$ field), the category the product belongs to (main_cat field) and the set of frequently copurchased products (also_buy field). It should be noted that in this dataset, each node belongs to exactly one (main) Amazon category so that, together, these three fields allow recovery of a large, undirected, labeled graph, with products as nodes, categories as labels and edges representing frequent co-purchasing product pairs. For this data set, we leveraged the co-purchasing relation among products, to naturally extract undirected and unweighted, labeled graphs. In more detail, for each pair $\left(\ell_{1}, \ell_{2}\right)$ of Amazon main categories, we extracted the undirected subgraph induced by the subset of nodes of category $\ell_{1}\left(\ell_{2}\right)$ that have at least one neighbour from category $\ell_{2}\left(\ell_{1}\right)$. We did not consider graphs with fewer than 100 nodes. This way, we retrieved 299 subgraphs of two categories (colors), with sizes ranging between 103 and 33922 nodes. We extended and applied this procedure to triples $\left(\ell_{1}, \ell_{2}, \ell_{3}\right)$ and quadruples $\left(\ell_{1}, \ell_{2}, \ell_{3}, \ell_{4}\right)$ of labels, obtaining 1147 subgraphs of three categories (colors), with sizes ranging between 352 and 30135 nodes, and 1408 subgraphs of four categories (colors), with sizes ranging between 1521 and 30086 nodes.

Algorithms. We compared the performance of the following algorithms that for simplicity we describe in the two-colors scenario:

2-DFSG. The optimal 2-approximation algorithm (Algorithm 2) based on Goldberg's optimal algorithm for the densest subgraph problem [22], described in Section 3.

Spectral Algorithms. Following [27, 33] and Theorem 2.2, we ran a variety of eigenvector rounding algorithms. These are all variants of a modified version of the General Sweep Algorithm (Algorithm 1) used in the proof of Theorem 2.2 that sorts the entries of the main eigenvector of $M$ four times (instead of a single one) according to the following criteria: i) non-increasing; ii) non-decreasing; iii) non-increasing absolute values; iv.) non decreasing absolute values. With these premises, we consider the following spectral algorithms. The first two are just the modified version of Algorithm 1 with different choices for $M$, while PS and FPS perform a slightly modified sweep that always affords a fair solution.

Single Sweep (SS). This algorithm is simply (Algorithm 1), when all previously mentioned sorting criteria are used, with $M=A$ and $\Delta=0$.

Fair Single Sweep (FSS). It is the execution of SS, this time on matrix $\left(I-f f^{T}\right) A\left(I-f f^{T}\right)$ instead of $A$.

Paired Sweep (PS). Paired Sweep is a modification of SS in which the fairness constraint is satisfied by construction in each subgraph produced by the rounding algorithm. This is done by considering the subsets $V_{\text {Red }}$ and $V_{\text {Blue }}$ of the nodes, sorting each of them separately according to the values of the corresponding entries in the main eigenvector of $A$ and then, for each $s=1, \ldots, \min \left(\left|V_{\text {Red }}\right|,\left|V_{\text {Blue }}\right|\right)$ considering the candidate set of nodes of cardinality $2 s$ obtained by taking the first $s$ nodes from each ordered subset. For a pseudocode, we refer to Algorithm 3.

Fair Paired Sweep (FPS). It is the execution of PS, this time on matrix $\left(I-f f^{T}\right) A\left(I-f f^{T}\right)$ instead of $A$. 


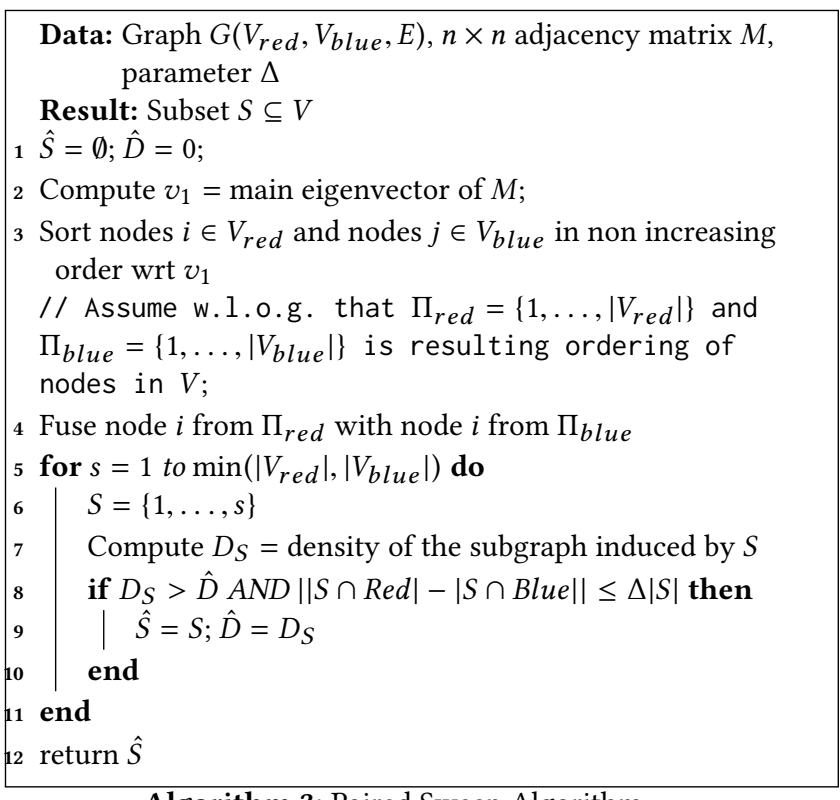

Algorithm 3: Paired Sweep Algorithm.

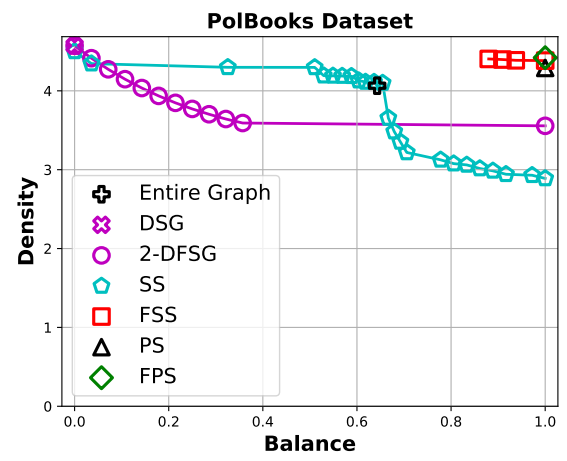

Figure 2: Pareto front of the subgraphs generated by each algorithm, w.r.t. density and balance, on PoLBooks dataset.

\subsection{Results}

Figure 2 shows the performance of our algorithms on PoLBooks dataset through the Pareto front of the subgraphs generated by each algorithm during its execution w.r.t. density and balance ${ }^{11}$. PS and FPS by construction only return fair solutions while the other algorithms potentially have trade-offs. In particular, 2-DSG (Algorithm 2) starts at the unconstrained optimum and proceeds to add nodes that increase balance while potentially decreasing density.

Figure 3 shows the distributions of the normalized density, over the entire set of AMAzON instances (for two, three and four colors), of the fair subgraphs retrieved by different algorithms. Normalization, performed to make solutions for different instances comparable, is done by scaling to the optimal density of the unconstrained

\footnotetext{
${ }^{11}$ Given two color classes Red and Blue, we define the balance of a subgraph containing $x$ Red and $y$ Blue nodes as $\min \left(\frac{x}{y}, \frac{y}{x}\right)$.
}

problem. ${ }^{12}$ Experimental results represented in Figure 3 (a, b, and c) show that spectral heuristics based on the paired-sweep technique (PS and FPS) consistently outperform 2-DFSG algorithm, despite its theoretical optimality (proved in a two-color scenario and in presence of a fair input graph), regardless the number of considered colors. In more detail, the FPS heuristic is the method that achieves the maximum median density. According to Figure 3 (b and c), it is evident that for a number of colors greater than two, the spectral methods that do not rely on the paired-sweep technique (SS and FS) are not the appropriate approaches for tackling the problem. Focussing on the two-colors scenario, depicted in Figure 3 (a), we have that, with the exception of SS which uses the original adjacency matrix and whose distribution is skewed toward lower density values, performances of spectral heuristics are comparable, with FPS achieving highest median density. Always in the two-colors scenario, we can observe that algorithms run on $\left(I-f f^{T}\right) A\left(I-f f^{T}\right)$ (FSS and FPS) respectively outperform their counterparts (SS and PS) run on $A$.

We report in Table 1 the percentage of instances each algorithm is not able to solve, i.e., for which it does not return a fair solution and, consequently, we assigned a density equal to 0 .

\begin{tabular}{|c|c|c|c|c|c|c|}
\hline \#Colors & \#Samples & SS & FSS & PS & FPS & 2-DFSG \\
\hline 2 & 299 & 0 & 0.33 & 0 & 0 & 3.01 \\
\hline 3 & 1147 & 73.93 & 95.55 & 0 & 0 & 5.31 \\
\hline 4 & 1408 & 92.54 & 99.64 & 0 & 0 & 1.91 \\
\hline
\end{tabular}

Table 1: Percentages of unfair solutions for Amazon dataset.

Data reported in Table 1 confirms the observation that spectral methods that do not rely on the paired-sweep technique essentially fail in recovering a dense fair subgraph in a context that involves more than two colors: the SS and FSS methods provided unfair solutions for almost all samples when the number of considered colors is greater than 2. As noted previously, PS and FPS cannot return unfair solutions: this is the reason behind the presence of zeros in their columns. It is worth to say that 2-DFSG (Algorithm 2) results in an unfair solution if the original graph is unbalanced and the unconstrained densest subgraph cannot be made fair via line 3. This justifies the presence of quantities greater than zero in the last column.

\begin{tabular}{|c|c|c|c|}
\hline AMAzoN dataset & 2 Colors & 3 Colors & 4 Colors \\
\hline \#Samples & 299 & 1147 & 1408 \\
\hline 2-DFSG & 46388.0101391 .3 & 151048.7152897 .9 & 127834.475275 .9 \\
\hline FPS & 359.8659 .2 & 1082.52073 .0 & 745.1524 .2 \\
\hline PS & 424.1842 .3 & 1130.22105 .8 & 775.3572 .0 \\
\hline FSS & 464.6860 .8 & 1652.42184 .6 & 1369.0983 .8 \\
\hline SS & 463.0858 .8 & 1664.82216 .3 & 1367.9986 .1 \\
\hline
\end{tabular}

Table 2: Average and standard deviation of the running times (in msec) of all proposed methods on AMAzon dataset: 2,3 and 4 colors.

\footnotetext{
${ }^{12}$ Hence, the maximum possible value on the $y$-axis is 1 .
} 


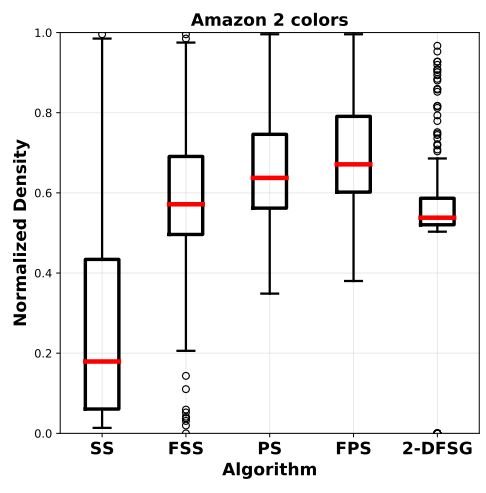

(a) AmAzon 2 colors: 299 subgraphs.

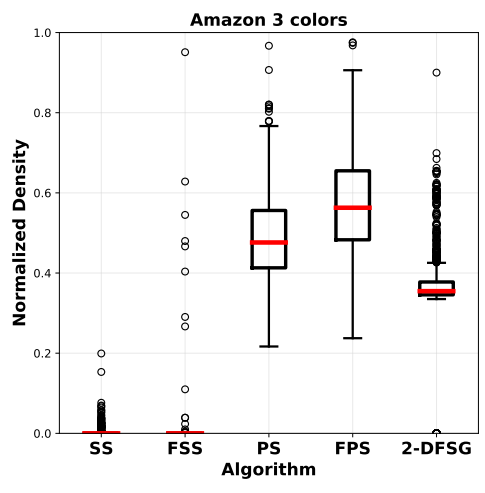

(b) AmAzon 3 colors: 1147 subgraphs.

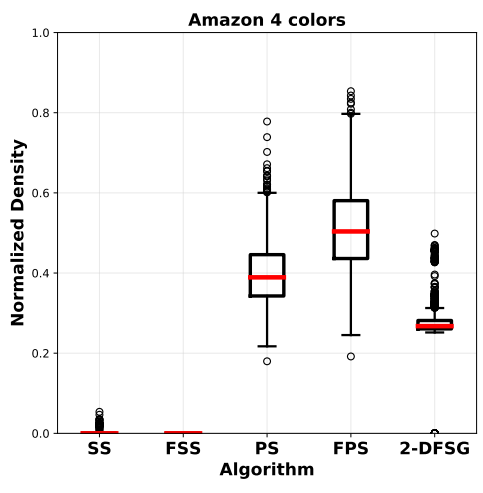

(c) AMAZon 4 colors: 1408 subgraphs.

Figure 3: Performance of our algorithms on AMAzon dataset for 2,3 and 4 colors on 299, 1147 and 1408 samples (subgraphs) respectively. Reported are aggregates over all generated subgraphs, with unfair solutions receiving a density of 0 , see Table 1.

Table 2 reports that spectral methods are faster than 2-DFSG. Indeed, the average running time of the 2-DFSG method is of two orders of magnitude greater than the one required by the spectral methods. This is coherent with the fact that the 2-DFSG method requires solving the Max-Flow problem, which is computationally expensive.

\begin{tabular}{|c|c|c|c|}
\hline AMAzoN dataset & 2 Colors & 3 Colors & 4 Colors \\
\hline \#Nodes, \#Edges & 1082301851733 & 1081851132578 & 1082201360241 \\
\hline 2-DFSG & 41260020.50 & 36189600.34 & 39913580.27 \\
\hline FPS & 361990.65 & 114670.45 & 319880.61 \\
\hline PS & 915820.56 & 393270.45 & 326430.50 \\
\hline FSS & 330740.51 & 17358 NoFairSol & 45465 NoFairSol \\
\hline SS & 264290.21 & 24161 NoFairSol & 32324 NoFairSol \\
\hline
\end{tabular}

Table 3: Running time (in msec) and solution quality (expressed as normalized density of the retrieved fair subgraph to the optimal density of the unconstrained problem) of all proposed methods on three AmAzon subgraphs with 2, 3 and 4 colors each. Each subgraph has roughly $100 \mathrm{~K}$ nodes and $1.1 \mathrm{M}$ edges.

Table 3 reports execution time and solution quality of all proposed methods on three not small-sized AmAzon subgraphs with 2,3 and 4 colors each. In particular, for what concerns the quality of the provided solutions, the results provided in Table 3 are completely in line with with the information extracted from Figure 3 and Table 1. Relation among execution times are also in line with what provided in Table 2, moreover, we can see that on the considered instances (2, 3 and 4 colors, $100 \mathrm{~K}$ nodes and $1.1 \mathrm{M}$ edges) the 2-DFSG method requires slightly more than one hour of computation, against $91 \mathrm{sec}$ required by the paired spectral heuristics (PS and FPS). These results suggest that the spectral approaches are suitable for dealing with not small-sized graphs.

\section{CONCLUSION AND FUTURE WORK}

In this work, we studied graphs with an arbitrary 2-coloring. For these graphs, the densest fair subgraph problem consists of finding a subgraph with maximal induced degree under the condition that both colors occur equally often. We observed that the problem is closely related to the densest at most $\mathrm{k}$ subgraph problem and thus has similar strong inapproximability results. On the positive side, we presented an optimal approximation algorithm under the assumption that the graph itself is fair, and a more involved spectral recovery algorithm inspired by the work of [28] on stochastic block models. In practice, the spectral recovery algorithm tended to dominate the approximation algorithm. We interpret these results as showing that (1) an approximation algorithm may not be the correct way to attack this problem, and (2) as previous work also suggests $[28,41]$, spectral relaxations seem to be an inexpensive tool to improve the fairness of algorithms geared towards recovery and learning.

Future work might consider extending this approach to more involved fairness constraints with provable guarentees. Empirically, we already observed that the spectral algorithms retain a good behaviour, while both theoretically and empirically, the performance of the approximation algorithm deteriorates. We identify two key problems that may be more manageable. First, one might consider the case where the graph only has to colors, but the colors may overlap, i.e. a node can be both red and blue. Clearly, the approximation results still hold in this case. Can one improve the analysis of spectral recovery scheme, depending on the degree of overlap? Second, one might consider the case of multiple disjoint colors, each of equal size. Such considerations have been studied in clustering literature $[3,4,8,16]$. Is it possible to derive similar results for densest subgraph?

\section{REFERENCES}

[1] Backurs, A., Indyk, P., Onak, K., Schieber, B., Vakilian, A., ANd Wagner, T. Scalable fair clustering. In Proceedings of the 36th International Conference on Machine Learning, ICML 2019, 9-15 June 2019, Long Beach, California, USA (2019), pp. $405-413$. 
[2] Bahmani, B., Goel, A., And Munagala, K. Efficient primal-dual graph algorithms for mapreduce. In Algorithms and Models for the Web Graph - 11th International Workshop, WAW 2014, Beijing, China, December 17-18, 2014, Proceed ings (2014), pp. 59-78.

[3] Becchetti, L., Bury, M., Cohen-Addad, V., Grandoni, F., And Schwiegelshohn, C. Oblivious dimension reduction for $k$-means: beyond subspaces and the johnson-lindenstrauss lemma. In Proceedings of the 51st Annual ACM SIGACT Symposium on Theory of Computing, STOC 2019, Phoenix, AZ, USA, June 23-26, 2019. (2019), pp. 1039-1050.

[4] Bera, S. K., Chakrabarty, D., Flores, N., and Negahbani, M. Fair algorithms for clustering. In Advances in Neural Information Processing Systems 32: Annual Conference on Neural Information Processing Systems 2019, NeurIPS 2019, 8-14 December 2019, Vancouver, BC, Canada (2019), pp. 4955-4966.

[5] Bercea, I. O., Gross, M., Khuller, S., Kumar, A., Rösner, C., Schmidt, D. R., AND SchmidT, M. On the cost of essentially fair clusterings. CoRR abs/1811.10319 (2018)

[6] Bhaskara, A., Charikar, M., Chlamtac, E., Feige, U., and Vijayaraghavan, A Detecting high log-densities: an $O\left(n^{1 / 4}\right)$ approximation for densest $k$-subgraph. In Proceedings of the 42nd ACM Symposium on Theory of Computing, STOC 2010 Cambridge, Massachusetts, USA, 5-8 fune 2010 (2010), pp. 201-210.

[7] Bhaskara, A., Charikar, M., Vijayaraghavan, A., Guruswami, V., and Zhou, Y. Polynomial integrality gaps for strong SDP relaxations of densest $k$-subgraph. In Proceedings of the Twenty-Third Annual ACM-SIAM Symposium on Discrete Algorithms, SODA 2012, Kyoto, Japan, January 17-19, 2012 (2012), pp. 388-405.

[8] Böhm, M., FAzzone, A., Leonardi, S., AND Schwiegelshohn, C. Fair clustering with multiple colors. CoRR abs/2002.07892 (2020).

[9] Boxell, L., Gentzkow, M., AND Shapiro, J. Is the internet causing politica polarization? evidence from demographics. Tech. rep., National Bureau of Economic Research, mar 2017.

[10] Celis, L. E., Huang, L., And Vishnoi, N. K. Multiwinner voting with fairness constraints. In Proceedings of the Twenty-Seventh International foint Conference on Artificial Intelligence, IfCAI 2018, July 13-19, 2018, Stockholm, Sweden. (2018), pp. $144-151$.

[11] Celis, L. E., Straszak, D., And Vishnoi, N. K. Ranking with fairness constraints. In 45th International Colloquium on Automata, Languages, and Programming, ICALP 2018, July 9-13, 2018, Prague, Czech Republic (2018), pp. 28:1-28:15.

[12] Chakraborty, T., Dalmia, A., Mukherjee, A., and Ganguly, N. Metrics for community analysis: A survey. ACM Comput. Surv. 50, 4 (2017), 54:1-54:37

[13] Charikar, M. Greedy approximation algorithms for finding dense components in a graph. In Approximation Algorithms for Combinatorial Optimization, Third International Workshop, APPROX 2000, Saarbrücken, Germany, September 5-8, 2000, Proceedings (2000), pp. 84-95.

[14] Chierichetti, F., Kumar, R., Lattanzi, S., and Vassilvitski, S. Fair clustering through fairlets. In Proceedings of the 30th Annual Conference on Neural Information Processing Systems (NIPS) (2017), pp. 5036-5044.

[15] Esfandiari, H., Hajiaghayi, M., AND Woodruff, D. P. Brief announcement Applications of uniform sampling: Densest subgraph and beyond. In Proceedings of the 28th ACM Symposium on Parallelism in Algorithms and Architectures, SPAA 2016, Asilomar State Beach/Pacific Grove, CA, USA, fuly 11-13, 2016 (2016), pp. $397-$ 399.

[16] Feldman, D., Schmidt, M., And Sohler, C. Turning big data into tiny data: Constant-size coresets for $k$-means, PCA and projective clustering. In Proceedings of the Twenty-Fourth Annual ACM-SIAM Symposium on Discrete Algorithms, SODA 2013, New Orleans, Louisiana, USA, fanuary 6-8, 2013 (2013), pp. 1434-1453.

[17] Feldman, M., Friedler, S. A., Moeller, J., Scheidegger, C., and VenkatasubRAMANIAN, S. Certifying and removing disparate impact. In Proceedings of the 21th ACM SIGKDD International Conference on Knowledge Discovery and Dato Mining (2015), pp. 259-268.

[18] Fratkin, E., Naughton, B. T., Brutlag, D. L., and Batzoglou, S. Motifcut regulatory motifs finding with maximum density subgraphs. Bioinformatics 22 14 (2006), e150-e157.

[19] Galimberti, E., Bonchi, F., And Gullo, F. Core decomposition and densest subgraph in multilayer networks. In Proceedings of the 2017 ACM on Conference on Information and Knowledge Management, CIKM 2017, Singapore, November 06 - 10, 2017 (2017), pp. 1807-1816.

[20] Gibson, D., Kumar, R., And Tomkins, A. Discovering large dense subgraphs in massive graphs. In Proceedings of the 31st International Conference on Very Large Data Bases, Trondheim, Norway, August 30 - September 2, 2005 (2005), pp. 721-732.

[21] Gionis, A., Junoueira, F., Leroy, V., Serafini, M., And Weber, I. Piggybacking on social networks. Proceedings of the VLDB Endowment 6, 6 (2013), 409-420.

[22] Goldberg, A. V. Finding a maximum density subgraph. Tech. Rep. UCB/CSD 84-171, EECS Department, University of California, Berkeley, 1984.

[23] Hardt, M., Price, E., AND Srebro, N. Equality of opportunity in supervised learning. In Advances in Neural Information Processing Systems 29: Annual Conference on Neural Information Processing Systems 2016, December 5-10, 2016, Barcelona, Spain (2016), pp. 3315-3323.

[24] Horwitz, S. The compositional impact of team diversity on performance:
Theoretical considerations. Human Resource Development Review 4 (06 2005), 219-245.

[25] Hu, S., Wu, X., AND Chan, T. H. Maintaining densest subsets efficiently in evolving hypergraphs. In Proceedings of the 2017 ACM on Conference on Information and Knowledge Management, CIKM 2017, Singapore, November 06 - 10, 2017 (2017), pp. 929-938.

[26] Huang, L., Jiang, S. H., And Vishnoi, N. K. Coresets for clustering with fairness constraints. In Advances in Neural Information Processing Systems 32: Annual Conference on Neural Information Processing Systems 2019, NeurIPS 2019, 8-14 December 2019, Vancouver, BC, Canada (2019), H. M. Wallach, H. Larochelle, A. Beygelzimer, F. d'Alché-Buc, E. B. Fox, and R. Garnett, Eds., pp. 7587-7598.

[27] Kannan, R., AND Vinay, V. Analyzing the structure of large graphs. Rheinische Friedrich-Wilhelms-Universität Bonn Bonn, 1999.

[28] Kleindessner, M., Samadi, S., Awasthi, P., and Morgenstern, J. Guarantees for spectral clustering with fairness constraints. In Proceedings of the 36th International Conference on Machine Learning, ICML 2019, 9-15 fune 2019, Long Beach, California, USA (2019), pp. 3458-3467.

[29] Krebs, V. Polbook-network-dataset, v. krebs, unpublished. http://www.orgnet. $\mathrm{com} /$.

[30] Lappas, T., Liu, K., AND Terzi, E. Finding a team of experts in social networks. In Proceedings of the 15th ACM SIGKDD International Conference on Knowledge Discovery and Data Mining (2009), KDD '09, pp. 467-476.

[31] MANurAngsi, P. Almost-polynomial ratio eth-hardness of approximating densest $\mathrm{k}$-subgraph. In Proceedings of the 49th Annual ACM SIGACT Symposium on Theory of Computing, STOC 2017, Montreal, QC, Canada, June 19-23, 2017 (2017), pp. 954961.

[32] Marcolino, L. S., JiAng, A. X., And TAmbe, M. Multi-agent team formation: diversity beats strength? In Twenty-Third International foint Conference on Artificial Intelligence (2013).

[33] MCSherry, F. Spectral partitioning of random graphs. In 42nd Annual Symposium on Foundations of Computer Science, FOCS 2001, 14-17 October 2001, Las Vegas, Nevada, USA (2001), pp. 529-537.

[34] Musco, C., Musco, C., and Tsourakakis, C. E. Minimizing polarization and disagreement in social networks. In Proceedings of the 2018 World Wide Web Conference on World Wide Web, WWW 2018, Lyon, France, April 23-27, 2018 (2018), pp. 369-378.

[35] Nasir, M. A. U., Gionis, A., Morales, G. D. F., and Girdzijauskas, S. Fully dynamic algorithm for top- $k$ densest subgraphs. In Proceedings of the 2017 ACM on Conference on Information and Knowledge Management, CIKM 2017, Singapore, November 06 - 10, 2017 (2017), pp. 1817-1826

[36] Ni, J., LI, J., AND MCAulEy, J. Justifying recommendations using distantlylabeled reviews and fine-grained aspects. In Proceedings of the 2019 Conference on Empirical Methods in Natural Language Processing and the 9th International foint Conference on Natural Language Processing (EMNLP-IFCNLP) (Hong Kong, China, Nov. 2019), Association for Computational Linguistics, pp. 188-197.

[37] Noriega-Campero, A., Bakker, M., Garcia-Bulle, B., And Pentland, A. Active fairness in algorithmic decision making. CoRR abs/1810.00031 (2018).

[38] Raghavendra, P., ANd Steurer, D. Graph expansion and the unique games conjecture. In Proceedings of the 42nd ACM Symposium on Theory of Computing, STOC 2010, Cambridge, Massachusetts, USA, 5-8 June 2010 (2010), pp. 755-764.

[39] Rösner, C., AND Schmidt, M. Privacy preserving clustering with constraints. In 45th International Colloquium on Automata, Languages, and Programming, ICALP 2018, July 9-13, 2018, Prague, Czech Republic (2018), pp. 96:1-96:14.

[40] Rozenshtein, P., Bonchi, F., Gionis, A., Sozio, M., And Tatti, N. Finding events in temporal networks: Segmentation meets densest-subgraph discovery. In IEEE International Conference on Data Mining, ICDM 2018, Singapore, November 17-20, 2018 (2018), pp. 397-406.

[41] Samadi, S., Tantipongpipat, U. T., Morgenstern, J. H., Singh, M., and VemPALA, S. The price of fair PCA: one extra dimension. In Advances in Neural Information Processing Systems 31: Annual Conference on Neural Information Processing Systems 2018, NeurIPS 2018, 3-8 December 2018, Montréal, Canada. (2018), pp. 10999-11010.

[42] Schmidt, M., Schwiegelshohn, C., ANd Sohler, C. Fair coresets and streaming algorithms for fair k-means. In Approximation and Online Algorithms - 17th International Workshop, WAOA 2019, Munich, Germany, September 12-13, 2019, Revised Selected Papers (2019), pp. 232-251.

[43] Sozio, M., AND Gionis, A. The community-search problem and how to plan a successful cocktail party. In Proceedings of the 16th ACM SIGKDD International Conference on Knowledge Discovery and Data Mining, Washington, DC, USA, July 25-28, 2010 (2010), pp. 939-948.

[44] Tantipongpipat, U., Samadi, S., Singh, M., Morgenstern, J. H., and Vempala, S. S. Multi-criteria dimensionality reduction with applications to fairness. In Advances in Neural Information Processing Systems 32: Annual Conference on Neural Information Processing Systems 2019, NeurIPS 2019, 8-14 December 2019, Vancouver, BC, Canada (2019), H. M. Wallach, H. Larochelle, A. Beygelzimer, F. d'Alché-Buc, E. B. Fox, and R. Garnett, Eds., pp. 15135-15145.

[45] Thanh, B. L., Ruggieri, S., And Turini, F. k-nn as an implementation of situation testing for discrimination discovery and prevention. In Proceedings of the 17th 
ACM SIGKDD International Conference on Knowledge Discovery and Data Mining (2011), pp. 502-510.

[46] Tsourakakis, C. E., Bonchi, F., Gionis, A., Gullo, F., and Tsiarli, M. A Denser than the densest subgraph: extracting optimal quasi-cliques with quality guarantees. In The 19th ACM SIGKDD International Conference on Knowledge Discovery and Data Mining, KDD 2013, Chicago, IL, USA, August 11-14, 2013 (2013), pp. $104-112$.

[47] Tsourakakis, C. E., Bonchi, F., Gionis, A., Gullo, F., and Tsiarl, M. A. Denser than the densest subgraph: extracting optimal quasi-cliques with quality guarantees. In The 19th ACM SIGKDD International Conference on Knowledge Discovery and Data Mining, KDD 2013, Chicago, IL, USA, August 11-14, 2013 (2013), pp. 104-112.

[48] Zafar, M. B., Valera, I., Gomez Rodriguez, M., AND Gummadi, K. P. Fairness beyond disparate treatment \& disparate impact: Learning classification without disparate mistreatment. In Proceedings of the 26th International Conference on World Wide Web (WWW) (2017), pp. 1171-1180.

[49] Zafar, M. B., Valera, I., Gomez-Rodriguez, M., and Gummadi, K. P. Fairness constraints: Mechanisms for fair classification. In Proceedings of the 20th International Conference on Artificial Intelligence and Statistics, AISTATS 2017, 20-22 April 2017, Fort Lauderdale, FL, USA (2017), pp. 962-970.

[50] Zafar, M. B., Valera, I., Gomez-Rodriguez, M., Gummadi, K. P., ANd Weller, A. From parity to preference-based notions of fairness in classification. In Advances in Neural Information Processing Systems 30: Annual Conference on Neural Information Processing Systems 2017, 4-9 December 2017, Long Beach, CA, USA (2017), pp. 228-238. 